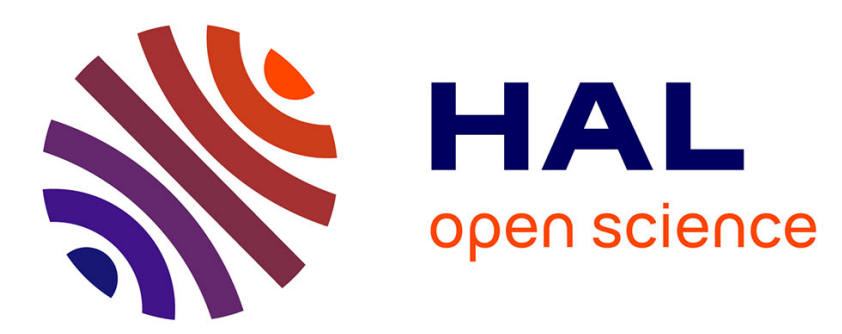

\title{
Démixtion et décomposition d'alliages semi-précieux quaternaires par oxydation à haute température: mécanismes diffusionnels
}

\author{
R. Charnay, B. Soegijono, P. Guiraldenq
}

\section{To cite this version:}

R. Charnay, B. Soegijono, P. Guiraldenq. Démixtion et décomposition d'alliages semi-précieux quaternaires par oxydation à haute température: mécanismes diffusionnels. Journal de Physique IV Proceedings, 1993, 03 (C9), pp.C9-159-C9-166. 10.1051/jp4:1993914 • jpa-00252351

\section{HAL Id: jpa-00252351 https://hal.science/jpa-00252351}

Submitted on 1 Jan 1993

HAL is a multi-disciplinary open access archive for the deposit and dissemination of scientific research documents, whether they are published or not. The documents may come from teaching and research institutions in France or abroad, or from public or private research centers.
L'archive ouverte pluridisciplinaire $\mathbf{H A L}$, est destinée au dépôt et à la diffusion de documents scientifiques de niveau recherche, publiés ou non, émanant des établissements d'enseignement et de recherche français ou étrangers, des laboratoires publics ou privés. 


\title{
Démixtion et décomposition d'alliages semi-précieux quaternaires par oxydation à haute température: mécanismes diffusionnels
}

\author{
R. Charnay, B. Soegijono et P. Guiraldenq
}

Ecole Centrale de Lyon, Département Matériaux, Mécanique-Physique, URA CNRS 447, B.P. 163, 69131 Ecully Cedex, France

\begin{abstract}
Résumé . - Les auteurs ont étudié les mécanismes structuraux et diffusionnels intervenant lors de l'oxydation à haute température sur deux familles d'alliages dentaires semi-précieux $(30 \%<$ $\mathrm{Ag}<60 \% ; 30 \%<\mathrm{Pd}<60 \% ; 5 \% \mathrm{Sn} ; 5 \% \mathrm{Cu})$ et $(30 \%<\mathrm{Au}<60 \% ; 30 \%<\mathrm{Pd}<60 \% ; 5 \%$ $\mathrm{Sn} ; 5 \% \mathrm{Cu})$. Ils ont pu montrer en particulier le rôle important des éléments nobles ( $\mathrm{Au}, \mathrm{Ag}, \mathrm{Pd})$ sur la cinétique d'oxydation, ainsi que sur la morphologie des couches d'oxydes. Linfluence de la composition chimique sur les différents mécanismes a été étudiée simultanément par diffraction $\mathrm{X}, \mathrm{ESCA}$ et MEB
\end{abstract}

\begin{abstract}
The authors have studied the structural mechanisms and the diffusion wich take place during high temperature oxidation of semi-precious dental alloys $(30 \%<\mathrm{Ag}<60 \%$; $30 \%<\mathrm{Pd}<60 \% ; 5 \% \mathrm{Sn} ; 5 \% \mathrm{Cu})$ and $(30 \%<\mathrm{Au}<60 \% ; 30 \%<\mathrm{Pd}<60 \% ; 5 \% \mathrm{Sn}$; $5 \% \mathrm{Cu}$ ). Particularly they have shown the important role played by noble metals ( $\mathrm{Au}, \mathrm{Ag}, \mathrm{Pd})$ on the oxidation kinetic and oxides morphology. The influence of chemical alloying effects have been studied by X-ray diffraction, ESCA and SEM.
\end{abstract}

\section{Introduction.}

De nombreuses recherches ont été entreprises ces dernières années sur des alliages semiprécieux (Pd-Ag) pour des applications odontologiques dans le but d'améliorer la qualité de la liaison céramo-métallique. Il a été montré qu'une oxydation préalable améliorait considérablement la tenue mécanique du couple métal-céramique [1-3]. Toutefois, les mécanismes réactionnels intervenant lors de l'oxydation restent mal connus. C'est pourquoi est ici étudié le rôle joué par des éléments facilement oxydables tels que le cuivre et l'étain sur les cinétiques d'oxydation et ceci dans des alliages base palladium-argent et palladium-or. La composition de ces alliages donnée dans le tableau I a été choisie de manière à encadrer celle des alliages utilisés industriellement, que nous prendrons comme référence, tout en conservant constants les éléments mineurs $\mathrm{Cu}$ et $\mathrm{Sn}$ qui sont indispensables pour la liaison. 
Tableau I. - Composition des alliages étudiés (\% pds).

\begin{tabular}{|l|c|c|c|c|c|c|c|c|}
\hline & $\mathrm{Pd}$ & $\mathrm{Ag}$ & $\mathrm{Au}$ & $\mathrm{Cu}$ & $\mathrm{Sn}$ & $\mathrm{In}$ & $\mathrm{Ga}$ & $\mathrm{Ru}$ \\
\hline Alliage 1 & 60 & 30 & & 5 & 5 & & & \\
\hline Alliage 2 & 55 & 35 & & 5 & 5 & & & \\
\hline Alliage 3 & 45 & 45 & & 5 & 5 & & & \\
\hline Alliage 4 & 30 & 60 & & 5 & 5 & & & \\
\hline Alliage 5 & 60 & & 30 & 5 & 5 & & & \\
\hline Alliage 6 & 45 & & 45 & 5 & 5 & & & \\
\hline Alliage 7 & 30 & & 60 & 5 & 5 & & & \\
\hline Alliage industriel DG 73 & 60,5 & 27,5 & & & 3 & 7 & 1,5 & 4 \\
\hline
\end{tabular}

\section{Matériaux et méthodes expérimentales.}

2.1 Alliages ÉTudiés. - Ces alliages ont été élaborés puis laminés par la Société Durant Girard à Lyon. Nous avons utilisé des plaquettes de $10 \times 10 \times 1 \mathrm{~mm}$ pour les caractérisations microstructurales et physicochimiques. Tous les échantillons ont subi préalablement un traitement thermique d'homogéneisation à $980^{\circ} \mathrm{C}$ pendant 24 heures dans des ampoules scellées sous vide, suivi d'un refroidissement rapide à l'eau.

2.2 MÉTHODES EXPÉRIMENTALES. - Les alliages ont été soigneusement polis à l'alumine et ensuite oxydés à $980^{\circ} \mathrm{C}$ à l'air, pendant des temps variant de $7 \mathrm{mn}$ à 18 heures. Les différentes cinétiques d'oxydation $\Delta m=f(t)$ ont été déterminées par thermogravimétrie. Nous avons pu également mesurer les profondeurs oxydées en fonction du temps par métallographie classique. Le réactif utilisé pour révéler la structure est un mélange équivolumique à $10 \%$ de cyanure de potassium et $10 \%$ de persulfate d'ammonium dans de l'eau. Les couches oxydées ont été caractérisées également par MEB, SDL, ESCA, microanalyse et diffraction X afin de mieux corréler les différentes cinétiques d'oxydation aux processus diffusionnels mis en jeu. A ce sujet, nous verrons plus loin que les cinétiques d'oxydation dépendent étroitement de la composition de l'alliage de base et de la nature des oxydes.

\section{Résultats.}

3.1 EXAMENS MÉTALLOGRAPHIQUeS EN COUPE. - Ces examens ont permis de mettre en évidence les différents processus d'oxydation intervenant dans les alliages:

1) soit par oxydation interne, uniquement en volume, comme dans le cas de l'alliage 1 (Fig. 1);

2) soit par oxydation interne mais cette fois en volume et aux joints de grains, comme pour les alliages 2-5-6-7 (Fig. 2) ;

3) soit enfin par oxydation interne associée à une oxydation externe, pour des temps longs de maintien en température: cas des alliages 3 et 4 (Fig. 3). 


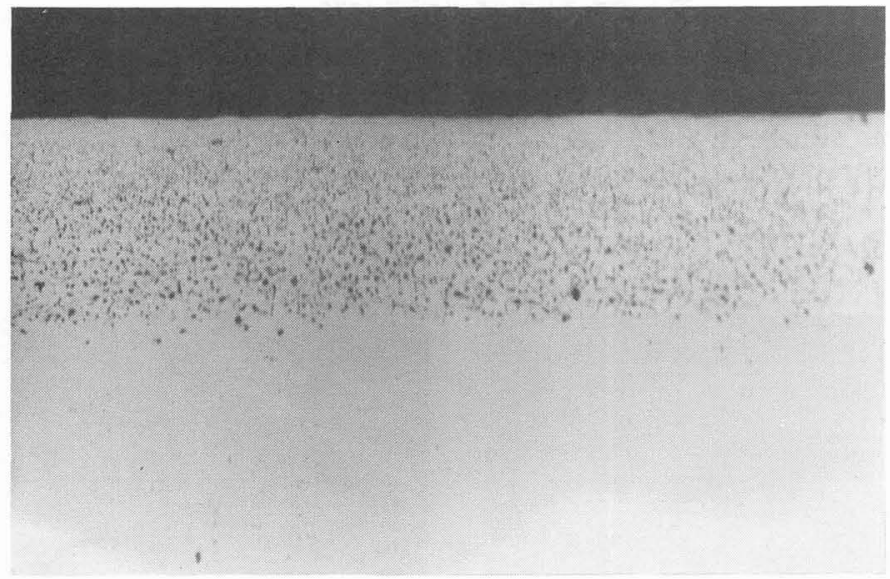

Fig. 1. - Alliage $1(60 \% \mathrm{Pd}, 30 \% \mathrm{Ag})$ oxydé 18 heures $(G=200)$.

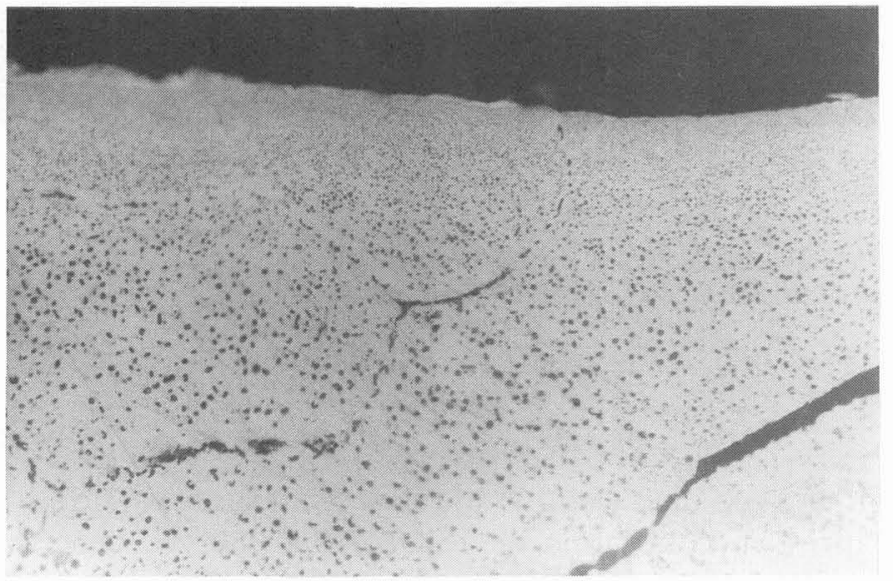

Fig. 2. - Alliage $2(55 \% \mathrm{Pd}, 35 \% \mathrm{Ag})$ oxydé 18 heures $(G=500)$.

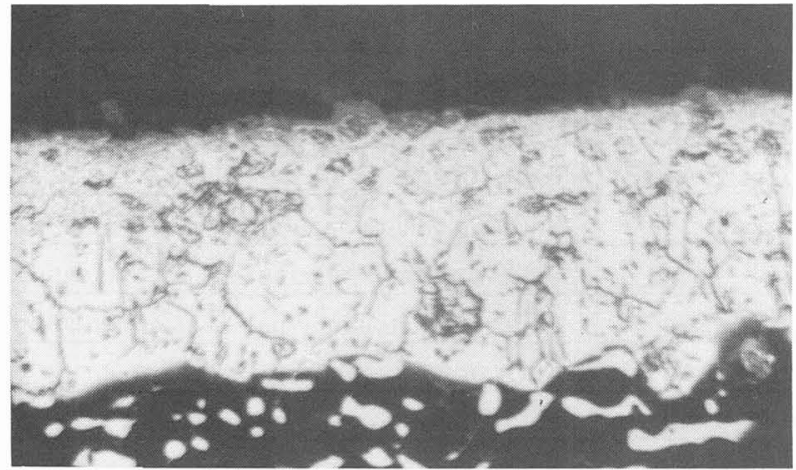

Fig. 3. - Alliage $4(30 \% \mathrm{Pd}, 60 \% \mathrm{Ag})$ oxydé 1 heure $(G=500)$. 
3.2 OBSERVATIONS DE LA SURFACE APRÈS OXYDATION (MEB). - Les différents faciès observés montrent que l'oxydation peut engendrer dans certains cas des porosités dans la couche oxydée comme par exemple pour les alliages 1 et 5 (Fig. 4) ou des décohésions intergranulaires dans le cas des alliages 2-6-7 (Fig. 5). Comme nous le verrons, ces différents défauts de structure dans les couches superficielles augmentent la vitesse d'oxydation.

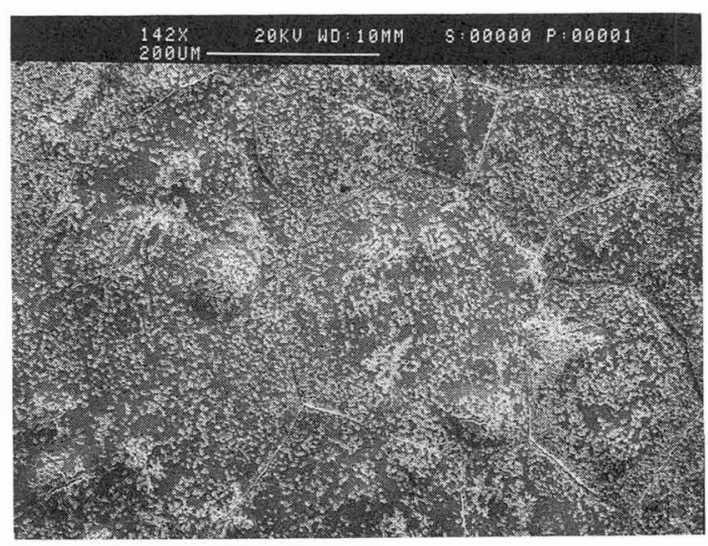

Fig. 4.

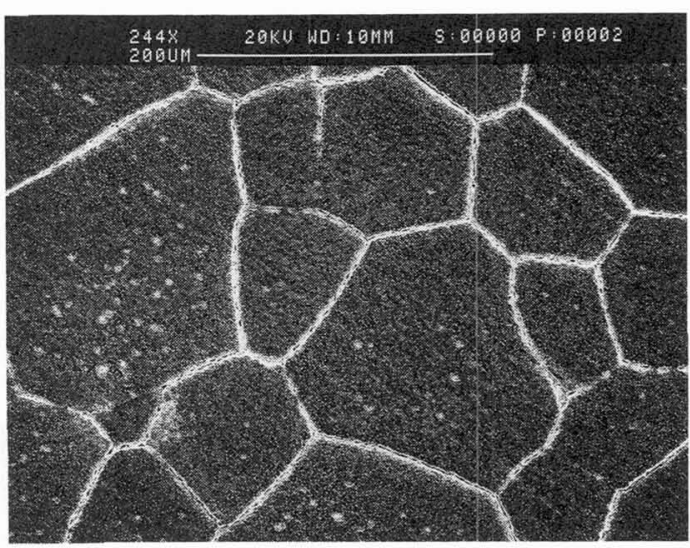

Fig. 5.

Fig. 4. - Alliage $5(60 \% \mathrm{Pd}, 30 \% \mathrm{Au})$ oxydé 18 heures.

Fig. 5. - Alliage $7(30 \% \mathrm{Pd}, 60 \% \mathrm{Au})$ oxydé 18 heures.

3.3 ANALYSES DES PHASES OXYDÉES PAR DIFFRACTION X ET MICROANALYSE. - Ces analyses ont été effectuées après une oxydation de 18 heures à $980^{\circ} \mathrm{C}$. Le tableau II donne les différents types d'oxydes présents dans les couches superficielles.

Tableau II. - Nature des oxydes présents dans les couches superficielles.

\begin{tabular}{|l|l|}
\hline Alliages & Nature des oxydes présents dans les couches superficielles \\
\hline $1-60 \% \mathrm{Pd} 30 \% \mathrm{Ag}$ & $\mathrm{SnO}_{2}$ (oxydation interne) \\
\hline $2-55 \% \mathrm{Pd} 35 \% \mathrm{Ag}$ & $\mathrm{SnO}_{2}$ (oxydation interne) \\
\hline $3-45 \% \mathrm{Pd} 45 \% \mathrm{Ag}$ & $\mathrm{SnO} 2$ (oxydation interne) $+\mathrm{CuO}$ (oxydation externe) \\
\hline $4-30 \% \mathrm{Pd} 60 \% \mathrm{Ag}$ & $\mathrm{SnO}_{2}$ (oxydation interne) $+\mathrm{CuO}$ (oxydation externe) \\
\hline $5-60 \% \mathrm{Pd} 30 \% \mathrm{Au}$ & $\mathrm{SnO}_{2}$ (oxydation interne) \\
\hline $6-45 \% \mathrm{Pd} 45 \% \mathrm{Au}$ & $\mathrm{SnO}_{2}$ (oxydation interne) \\
\hline $7-30 \% \mathrm{Pd} 60 \% \mathrm{Au}$ & $\mathrm{SnO}_{2}$ (oxydation interne) \\
\hline DG 73 & $\mathrm{In}_{2} \mathrm{O}_{3}$ (oxydation interne) \\
\hline
\end{tabular}


Ces résultats montrent que la nature des oxydes formés est directement liée à la composition des alliages. Ceci confirme les travaux de J.R. Mackert [4-5] qui a mis en évidence la présence d'oxyde d'étain $\mathrm{SnO}_{2}$ dans un alliage $53,5 \% \mathrm{Pd}-37,5 \% \mathrm{Ag}-8,5 \% \mathrm{Sn}-0,5 \%$ In après oxydation entre 750 et $1010^{\circ} \mathrm{C}$. De même $\mathrm{H}$. Ohno [6-7] a identifié la présence de $\mathrm{SnO}_{2}, \mathrm{In}_{2} \mathrm{O}_{3}, \mathrm{Fe}_{2} \mathrm{O}_{3}$ dans des alliages industriels complexes $85,5 \% \mathrm{Au}-6 \% \mathrm{Pt}-6 \% \mathrm{Pd}-1 \%$ $\mathrm{Ag}-0,3 \%$ à $1,5 \%$ In - $0,3 \%$ à $1,5 \%$ Sn après une oxydation d'une heure à $1000^{\circ} \mathrm{C}$.

3.4 MESURES THERMOGRAVIMÉTRIQUES. - Les résultats correspondant à des essais réalisés à $980^{\circ} \mathrm{C}$ et pour des temps compris entre $7 \mathrm{~min}$ et 18 heures sont regroupés dans les figures 6 à 11 . La température d'oxydation de $980^{\circ} \mathrm{C}$ choisie ici correspond à celle réalisée industriellement pour la fabrication de prothèses céramométalliques.

L'ensemble de ces résultats montre que les cinétiques d'oxydation à $980^{\circ} \mathrm{C}$ de ces alliages suivent des lois classiques de diffusion en $\sqrt{t}$ d'une manière monotone sauf dans le cas de l'alliage 4 qui montre une anomalie thermogravimétrique pour des temps d'oxydation supérieurs à 4 heures. Ceci est dû à un changement de régime diffusionnel du fait de la création d'une couche externe importante de $\mathrm{CuO}$.

\section{Discussion des résultats.}

\subsection{EN CE QUi CONCERNE LES ALliageS SYNTHÉTIQUES Pd-Ag.}

1) le palladium ralentit les cinétiques d'oxydation (Figs. 6 et 10) par rapport à l'argent,

2) la teneur en palladium détermine le mode d'oxydation préférentiel. En effet, on a vu que l'oxydation d'alliages contenant 60 à $55 \% \mathrm{Pd}$ et 30 à $35 \% \mathrm{Ag}$ conduit à une précipitation interne de $\mathrm{SnO}_{2}$ (Tab. II). Au contraire, pour des teneurs plus faibles en palladium (45\% à $30 \% \mathrm{Pd}$ et $45 \%$ à $60 \% \mathrm{Ag}$ ) le mode d'oxydation devient mixte (oxydation externe avec formation de $\mathrm{GuO}$ et précipitation interne de $\mathrm{SnO}_{2}$ ),

3) l'oxydation intergranulaire associée à des contraintes thermomécaniques peuvent engendrer un déchaussement partiel des grains comme dans le cas de l'alliage 2 (Fig. 2)

4) en ce qui concerne l'alliage industriel DG 73 pris comme référence, on constate que l'oxydation entraine d'une part un précipitation interne de $\mathrm{In}_{2} \mathrm{O}_{3}$ et d'autre part une remontée en surface du palladium et de l'argent.

Ce phénomène a déjà été rencontré par J.R. Mackert [4-5] sur un alliage contenant $53,5 \% \mathrm{Pd}, 37,5 \% \mathrm{Ag}, 8,5 \% \mathrm{Sn}, 0,5 \% \mathrm{In}$. Selon cet auteur, cette démixtion serait liée aux contraintes internes naissant dans la couche oxydée au cours du traitement thermique suivant des mécanismes de fluage-diffusion évoqués par d'autres auteurs [8,9].

\subsection{EN CE QUi CONCERNE LES ALliaGeS Pd-Au.}

1) contrairement au cas précédent, le palladium augmente la cinétique d'oxydation (Fig. 7, 11). Celle ci est toutefois plus lente que pour les alliages Pd-Ag. Nous retrouvons ici l'effet favorable de l'or vis à vis de l'oxydation.

2) d'un point de vue microstructural, la diffusion de l'oxygène s'accompagne toujours de microporosités dans les couches oxydées pour des temps d'oxydation longs (Figs 4,5 ) quelle que soit la composition de l'alliage. Ceci peut être un facteur intéressant pour améliorer la qualité des liaisons céramo-métalliques. 


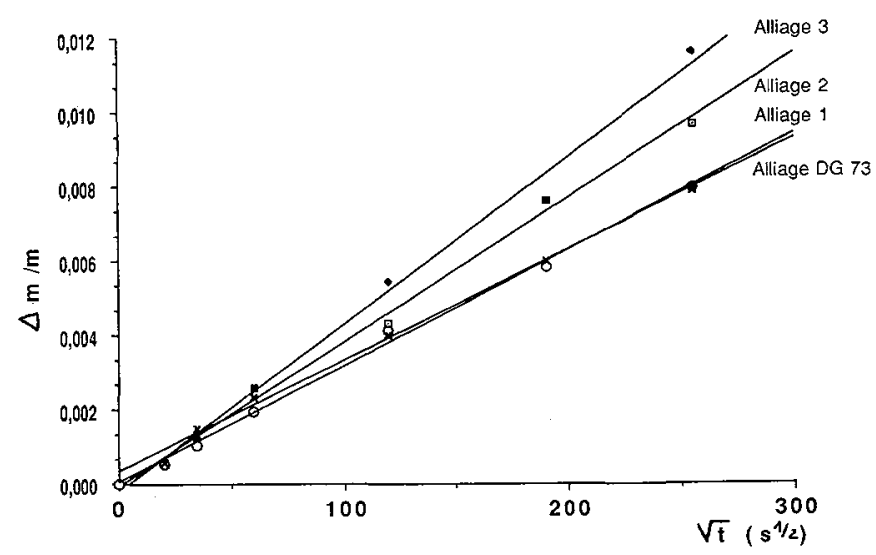

Fig. 6. - Evolution de $\Delta m / m$ en fonction de $\sqrt{t}$ (cas des alliages 1-2-3-DG73).

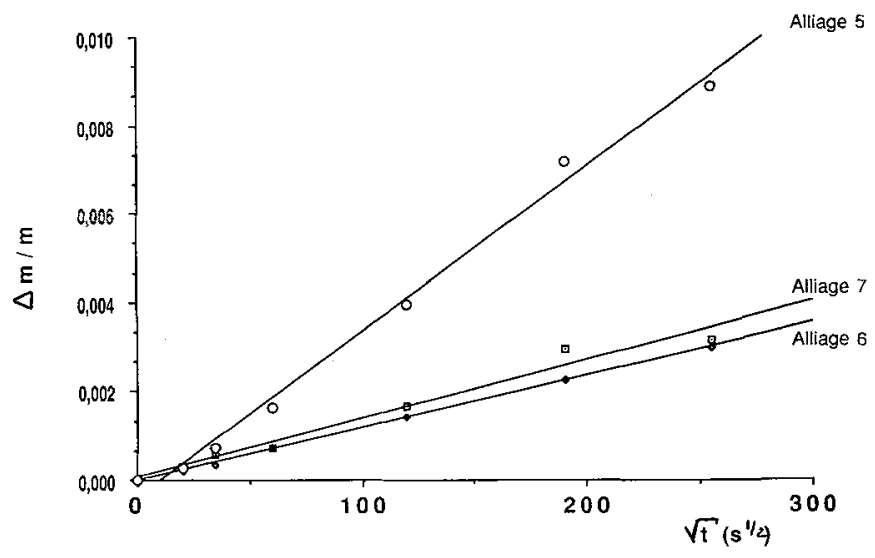

Fig. 7. - Evolution de $\Delta m / m$ en fonction de $\sqrt{t}$ (cas des alliages 5-6-7).

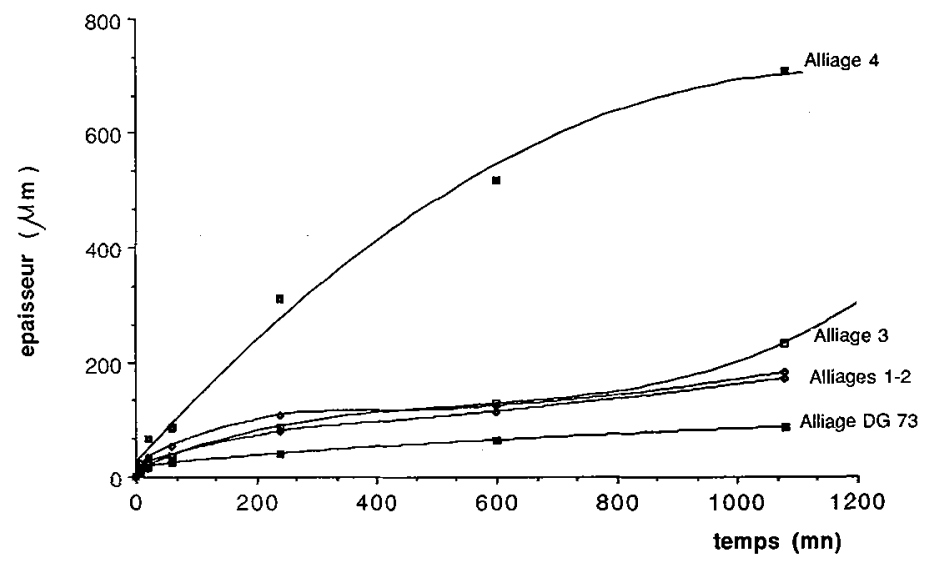

Fig. 8. - Variation de l'épaisseur oxydée en fonction du temps (alliages 1-2-3-4 et DG 73). 


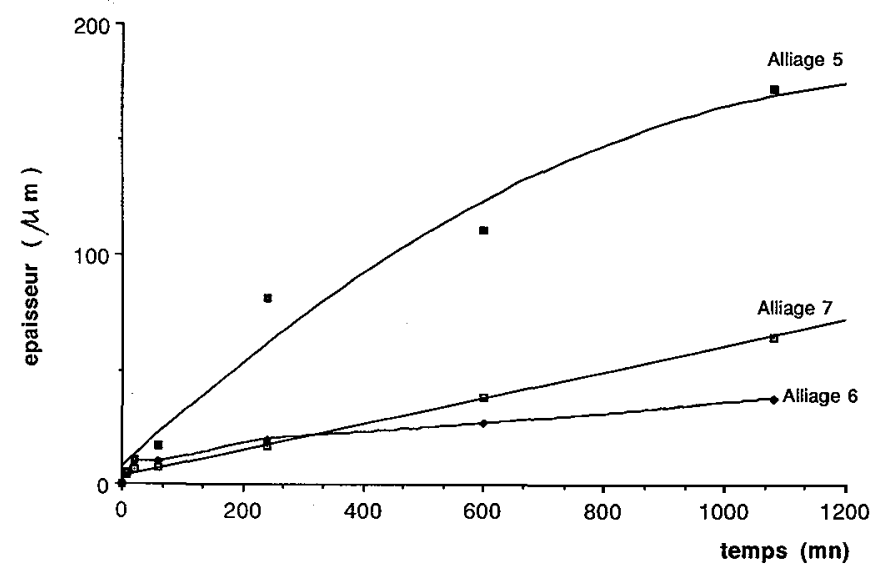

Fig. 9. - Variation de l'épaisseur oxydée en fonction du temps (alliages 5-6-7).

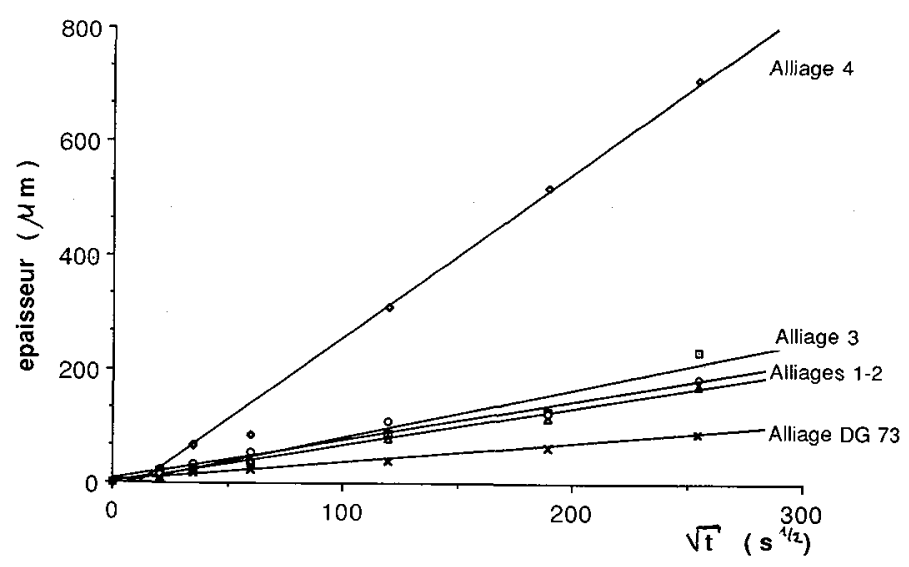

Fig. 10. - Variation de l'épaisseur oxydée avec $\sqrt{t}$ (alliages 1-2-3-4 et DG 73).

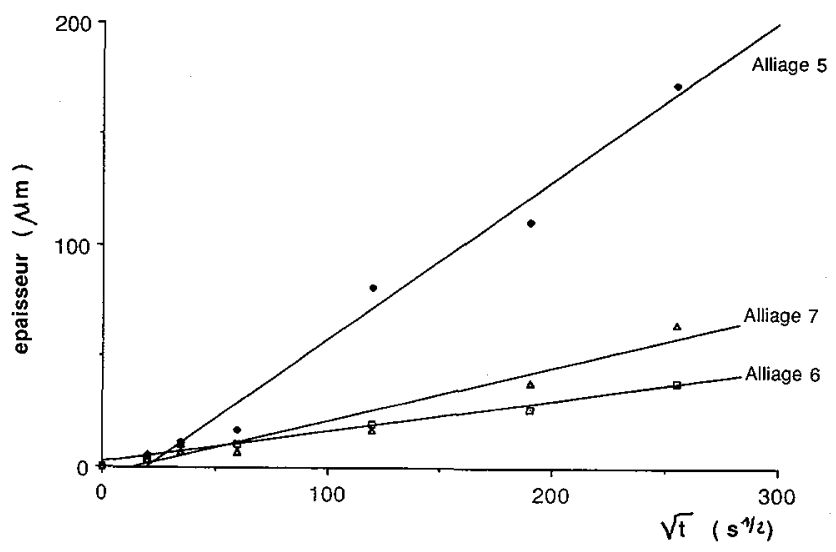

Fig. 11. - Variation de l'épaisseur oxydée avec $\sqrt{t}$ (alliages 5-6-7). 


\section{Conclusion.}

Par cette étude nous avons montré le rôle particulièrement important de la composition de l'alliage sur la cinétique d'oxydation ainsi que sur les différents modes d'oxydation. Ce travail sera prolongé d'une part par une analyse des energies d'activation liées aux processus d'oxydation et d'autre part par une caractérisation micromécanique (flexion 4 points) de l'interface métal-céramique.

\section{Bibliographie}

[1] Fairhurst C.W., Mackert J.R., Warren twiggs S., Ceram. Eng. Sci. Proc. 6 (1) (1983) 66-83.

[2] Hero H., Austrheim E., Dent. Mater. (3) (1987) 168-175.

[3] Shell J.S., NiElSEN J.P., J. Dent. Res. 41 (6) (1962) 1424-1437.

[4] MarCKert J.R., Metall. Trans. A 17A (1986) 746-749.

[5] MACKerT J.R., Ringle R.D., FAIRHUST C.W., J. Dent. Res. 62 (12) (1983) 1229-1235.

[6] Ohno H., MiYakawa O., Watanabe K., Shiokawa N., J. Dent. Res. 61 (11) (1982) 1255-1261.

[7] Ohno H., KanZaWa Y., Kawashima I., Shiokawa N., J. Dent. Res. 62 (6) (1983) 774779.

[8] GIFKINS R.C., LANGDON T.G., Scr. metall. 4 (1970) 563-567.

[9] Herring C., J. Appl. Phys. 21 (5) (1950) 437-445. 